\title{
Soluble intercellular adhesion molecule-1 as an early detection marker for radiation pneumonitis
}

\author{
Y. Ishii, S. Kitamura
}

Soluble intercellular adhesion molecule-1 as an early detection marker for radiation pneumonitis. Y. Ishii, S. Kitamura. C ERS Journals Ltd 1999.

ABSTRACT: To investigate the role of intercellular adhesion molecule-1 (ICAM-1) in the pathogenesis of radiation pneumonitis and to determine whether the measurement of soluble ICAM-1 (sICAM-1) levels is useful for predicting the onset of pneumonitis, the levels of sICAM-1 were measured in serum and bronchoalveolar lavage (BAL) fluids from patients with lung malignancy who received radiotherapy.

A total of 30 patients were irradiated with a total dose of $\sim 60 \mathrm{~Gy}$. Blood samples were taken before, midway and after radiotherapy. BAL was also performed before and after radiotherapy in seven cases. The sICAM-1 concentration was measured using an enzyme-linked immunosorbent assay kit with two different monoclonal antibodies.

Twelve out of 30 cases developed radiation pneumonitis (pneumonitis group), and the other cases did not (nonpneumonitis group). Serum levels of SICAM-1 after radiotherapy were significantly elevated in the pneumonitis group, but not in the nonpneumonitis group. In some of the cases in the pneumonitis group, sICAM-1 levels began to increase at an early phase of irradiation. In one case of pneumonitis in which BAL was performed, the total cell count and the number of lymphocytes increased markedly, as did the level of SICAM-1 in BAL fluid.

These findings suggest that intercellular adhesion molecule-1 may play an important role in the development of radiation pneumonitis and that soluble intercellular adhesion molecule-1 may be a useful marker for the early detection of radiation pneumonitis.

Eur Respir J 1999; 13: 733-738.

Radiation is one of the main therapies for lung cancer and other malignancies, but radiation-induced lung injury is a limiting factor for therapy and is sometimes lifethreatening. There are two types of radiation-induced lung injury: radiation pneumonitis and radiation fibrosis. In 5$15 \%$ of all patients who receive chest radiotherapy, early acute pneumonitis develops within 12 weeks of radiotherapy $[1,2]$, characterized by cough, fever, and mild dyspnoea. Pulmonary infiltration can be shown not only in the irradiated lung but also in the contralateral lung. The condition of these patients sometimes worsens, and the dyspnoea can be life-threatening. In contrast to radiation pneumonitis, radiation fibrosis occurs 6-12 months after radiotherapy in up to $75 \%$ of all irradiated patients and a well-defined area of fibrosis is confined to the field of irradiation $[1,2]$. Although the histological changes resulting from direct irradiation of the lung have been well documented, the mechanisms whereby limited unilateral irradiation produces symptoms suggestive of a severe generalized process have not yet been established. Pathological changes in radiation pneumonitis include hyaline membrane formation in the alveolar spaces, endothelial injury, destruction of type I and II cells and infiltration of the interstitial spaces by inflammatory cells [1].

Recently, studies using bronchoalveolar lavage (BAL) fluids have indicated that lymphocytic alveolitis can be
Dept of Pulmonary Medicine, Jichi Medical School, Tochigi, Japan.

Correspondence: Y. Ishii

Dept of Pulmonary Medicine

Jichi Medical School

Minamikawachi, Yakushiji

Tochigi 329-04

Japan

Fax: 81285443586

Keywords: Adhesion molecule bronchoalveolar lavage lymphocyte alveolitis radiation pneumonitis soluble intercellular adhesion molecule-1

Received: February 101998

Accepted after revision October 81998 observed in patients with radiation pneumonitis [2-4]. Histological studies based on open lung biopsies, taken from the irradiated lung fields, have also suggested lymphocytic infiltration as the dominant finding in acute-phase pneumonitis [5]. These findings suggest that lymphocyte recruitment and activation in the lung play an important role in the pathogenesis of radiation pneumonitis.

In the mechanism of leukocyte accumulation in the lungs, the mediation of adhesion molecules is essential. The intercellular adhesion molecule-1 (ICAM-1), a member of the immunoglobulin superfamily of adhesion molecules, is expressed on various cell surfaces, including vascular endothelial cells, epithelial cells and lymphocytes [6]. ICAM-1 is a ligand for the lymphocyte function-associated antigen-1 (LFA-1), which is found on lymphocytes and other leukocytes [7]. The ICAM-1/LFA1 interaction is important in T-lymphocyte activation and lymphocyte migration into inflammatory sites [8-11]. Recently, it has been reported that radiation induces the expression of ICAM-1 on lung cells and that ICAM-1 plays an important role in the pathogenesis of radiation-induced inflammation [12-16].

ICAM-1 shed from the cell surface exists in the blood and alveolar lining fluids in a soluble form [17]. Measurement of the soluble ICAM-1 (sICAM-1) has been reported to be useful in evaluating and monitoring disease 
activity in various immunological and inflammatory disorders [18-24].

To investigate the possible role of ICAM-1 in the pathogenesis of radiation pneumonitis accompanied by lymphocytic alveolitis and to determine whether the measurement of sICAM-1 levels is useful for predicting the onset of pneumonitis, the levels of sICAM-1 in serum and BAL fluid were measured in patients with lung malignancy who received radiotherapy.

\section{Materials and methods}

\section{Study population}

The protocol used in this study was reviewed and approved by the Jichi Medical School Institutional Review Board for Human Research and written informed consent was obtained from the subjects. In total, 30 patients with lung cancer or other lung malignancies, who received irradiation to the chest, were selected sequentially for this study. Patients with apparent infection, such as obstructive pneumonia, were excluded. All patients received hyperfractionation radiotherapy: 1.5-1.6 Gy twice a day, in 10 fractions.week ${ }^{-1}$. Patients were considered to have developed radiation pneumonitis if they presented with: 1) typical clinical symptoms such as cough, fever and dyspnoea, 2) an infiltrative shadow on the chest radiograph, and 3) a decrease in resting arterial oxygen tension $\left(\mathrm{Pa}_{\mathrm{a}} \mathrm{O}_{2}\right)$. Other causes of exacerbation, such as infection, lymphangitis and pulmonary infarction, were ruled out in all patients.

\section{Sample collection}

Blood samples were taken before, midway and after radiotherapy. If the patient developed radiation pneumonitis, an additional blood sample was taken at that time. The mean \pm SD duration between the end of radiotherapy and the onset of radiation pneumonitis was $27.8 \pm 37.7$ days. In the control group, additional blood samples were taken 1 month after radiotherapy.

BAL was also performed by the standard method, before and after radiotherapy, in seven patients who agreed to receive this procedure. Only one of these cases had radiation pneumonitis, while the other six did not. An irradiated segment which was not directly involved in lung cancer was lavaged with three $50-\mathrm{mL}$ aliquots of sterile saline. After filtration through two layers of gauze, a small aliquot was taken for the assessment of total cell counts, using a haemocytometer. A differential cell count was obtained by identifying over 500 cells on Diff-Quick stained cytocentrifuge preparations. After centrifugation $(250 \times g, 10 \mathrm{~min})$, the cell supernatant was frozen at $-20^{\circ} \mathrm{C}$. Aliquots were later thawed and analysed.

\section{Soluble intercellular adhesion molecule-1 and lactate dehydrogenase assay}

Concentrations of sICAM-1 in serum and BAL fluid were measured using an sICAM-1 enzyme-linked immunosorbent assay (ELISA) kit (T Cell Diagnostics, Cambridge, MA, USA). All samples were diluted 1:100 in diluent buffer prior to assay. Samples were added to microtitre plates precoated with an anti-ICAM-1 primary antibody. Then, horseradish peroxidase-conjugated antiICAM-1 secondary antibody, which recognizes another epitope on the ICAM-1 molecule, was added. After $2 \mathrm{~h}$ of incubation, the plates were washed and chromogen solution was added to the wells. The absorbance at $490 \mathrm{~nm}$ was determined using a microplate reader. The coefficient of variation for intra-assay and interassay variability was $<5 \%$. The normal ranges of sICAM- 1 in serum and in BAL fluid from healthy controls ( $\mathrm{n}=13$, age $28.3 \pm 4.0 \mathrm{yrs}$ ) were $243 \pm 49 \mathrm{ng} \cdot \mathrm{mL}^{-1}$ and $59 \pm 36 \mathrm{ng} \cdot \mathrm{mL}^{-1}$, respectively [18].

Serum lactate dehydrogenase (LDH) activity was also determined by the Wroblewski-LaDue method.

\section{Statistical analysis}

Results are expressed as the meansd. Differences between two group means were compared by the MannWhitney U-test. Multigroup comparisons were made by a one-way analysis of variance (ANOVA). Linear regression analysis was used to determine the correlation between investigated parameters. A p-value $<0.05$ was considered significant.

\section{Results}

\section{Profile of study population}

The profile of the patients is shown in tables 1 and 2 . Thirty-two patients were enrolled in this study, but two of them were excluded because they developed bacterial pneumonia during radiotherapy. Twelve out of 30 cases developed radiation pneumonitis (pneumonitis group) and the other cases did not (nonpneumonitis group). There was no significant difference in the age distribution $(64.9 \pm 6.7$ versus $66.1 \pm 10.7 \mathrm{yrs})$ or irradiated doses (56.3 \pm 5.9 versus $58.3 \pm 3.5 \mathrm{~Gy}$ ) between the two groups.

\section{Serum soluble intercellular adhesion molecule-1}

Serum levels of sICAM-1 before radiotherapy were significantly elevated in both the pneumonitis group (326 \pm $42 ; n=12)$ and the nonpneumonitis group $(312 \pm 26 ; n=$ 18) compared with normal values obtained from healthy volunteers $\left(243 \pm 49 \mathrm{ng} \cdot \mathrm{mL}^{-1}, \mathrm{n}=13\right)$. There was no difference between the two groups. Serum levels of sICAM-1 after radiotherapy were elevated in the pneumonitis group compared with the baseline levels before radiotherapy (fig. 1). In 10 out of 12 cases (83\%), serum levels of sICAM-1 were elevated at the onset point of pneumonitis. In seven out of 12 cases $(58 \%)$, they were elevated at the end-point of radiotherapy. Moreover, in four out of 12 cases (33\%), sICAM-1 levels began to increase at the midway point of irradiation. In contrast, serum levels of sICAM-1 did not increase throughout radiotherapy in the control group (fig. 1). Even at one month after radiotherapy, there was no increase in sICAM-1 levels.

The average sICAM-1 concentrations in serum for each group are shown in figure 2 . In the pneumonitis group, a significant elevation in sICAM-1 levels was seen at the end of the radiation therapy $\left(514 \pm 82 \mathrm{ng} \cdot \mathrm{mL}^{-1}\right)$ and at the point of onset of pneumonitis $\left(790 \pm 109 \mathrm{ng} \cdot \mathrm{mL}^{-1}\right) \mathrm{com}-$ pared with a baseline "before" value $\left(326 \pm 42 \mathrm{ng} \cdot \mathrm{mL}^{-1}\right.$; $\mathrm{p}<0.05)$. 
Table 1. - Characteristics of patients without radiation pneumonitis

\begin{tabular}{|c|c|c|c|c|c|c|}
\hline $\begin{array}{l}\text { Patient } \\
\text { No. }\end{array}$ & $\begin{array}{l}\text { Age } \\
\text { yrs }\end{array}$ & Sex & $\begin{array}{l}\text { Histological } \\
\text { type }\end{array}$ & $\begin{array}{l}\text { Preceding } \\
\text { therapy }\end{array}$ & $\begin{array}{l}\text { Radiation dose } \\
\text { Gy }\end{array}$ & BAL \\
\hline 1 & 78 & M & $\mathrm{Sm}$ & Chemo & 50 & \\
\hline 2 & 76 & $\mathrm{M}$ & $\mathrm{Sq}$ & Chemo & 60 & \\
\hline 3 & 66 & $\mathrm{M}$ & $\mathrm{Sq}$ & Chemo & 59 & \\
\hline 4 & 64 & $\mathrm{M}$ & $\mathrm{Sq}$ & $\mathrm{ND}$ & 59 & + \\
\hline 5 & 59 & M & $\operatorname{AdSq}$ & Chemo & 61 & + \\
\hline 6 & 46 & M & $\mathrm{Sm}$ & ND & 53 & \\
\hline 7 & 75 & $\mathrm{M}$ & $\mathrm{Sq}$ & Chemo & 60 & \\
\hline 8 & 68 & $\mathrm{M}$ & $\mathrm{Sq}$ & Chemo & 60 & + \\
\hline 9 & 64 & $\mathrm{M}$ & $\mathrm{Sm}$ & Chemo & 50 & + \\
\hline 10 & 65 & M & $\mathrm{Sq}$ & Op, Chemo & 60 & \\
\hline 11 & 77 & $\mathrm{M}$ & $\mathrm{Sq}$ & Chemo & 58 & \\
\hline 12 & 45 & $\mathrm{M}$ & Ad & Chemo & 60 & \\
\hline 13 & 79 & $\mathrm{M}$ & $\mathrm{Sq}$ & Op, Chemo & 60 & + \\
\hline 14 & 59 & M & $\mathrm{Sm}$ & Chemo & 59 & \\
\hline 15 & 76 & $\mathrm{M}$ & $\mathrm{Sq}$ & Chemo & 60 & \\
\hline 16 & 55 & M & Sarcoma & Chemo & 59 & \\
\hline 17 & 77 & M & Sarcoma & Chemo & 62 & \\
\hline 18 & 60 & $\mathrm{~F}$ & Ad & Chemo & 60 & + \\
\hline Mean \pm SD & $.1 \pm 10$ & & & & $58.3 \pm 3.5$ & \\
\hline
\end{tabular}

M: male; F: female; Sm: small cell carcinoma; Sq: squamous cell carcinoma; Ad: adenocarcinoma; ND: not done; Chemo: chemotherapy; Op: operation; BAL: bronchoalveolar lavage.

\section{Serum lactate dehydrogenase activity}

In both groups, serum LDH activity showed a tendency to decrease after radiation therapy. In seven out of 12 cases who developed pneumonitis, however, serum LDH levels increased only at the point of onset of pneumonitis (fig. 3). There was a significant correlation between the serum sICAM-1 level and LDH activity only at the point of onset of pneumonitis $(r=0.665, \mathrm{p}<0.05)$ (fig. 4).

\section{Bronchoalveolar lavage analysis}

BAL was performed before and after radiotherapy in six patients who did not develop radiation pneumonitis and in one case who developed pneumonitis. After radiotherapy, the total cell count and the percentage of lymphocytes in the BAL fluid increased in two out of six cases without pneumonitis. sICAM-1 levels in the BAL fluid also increased in four out of six cases. In the case with pneumonitis in which BAL was performed, the total cell count and the number of lymphocytes increased markedly (up to $90 \%$ ) and the level of sICAM-1 in BAL fluid also increased (fig. 5).

\section{Discussion}

Much evidence has been reported indicating that the sequestration of lymphocytes in the lungs is a characteristic pathological feature in radiation pneumonitis [2-4]. ROBERTS et al. [2] reported radiation pneumonitis cases with lymphocytic alveolitis which were detected by BAL and speculated that the pathogenesis of early generalized radiation pneumonitis is a lymphocyte-mediated hypersensitivity reaction. Antigen release occurs in the process

Table 2. - Characteristics of patients with radiation pneumonitis

\begin{tabular}{|c|c|c|c|c|c|c|c|c|}
\hline $\begin{array}{l}\text { Patient } \\
\text { No. }\end{array}$ & $\begin{array}{l}\text { Age } \\
\text { yrs }\end{array}$ & Sex & $\begin{array}{l}\text { Histological } \\
\text { type }\end{array}$ & $\begin{array}{l}\text { Preceding } \\
\text { therapy }\end{array}$ & $\begin{array}{l}\text { Radiation dose } \\
\text { Gy }\end{array}$ & $\begin{array}{c}\text { Interval between } \\
\text { radiotherapy and onset } \\
\text { days }\end{array}$ & BAL & Outcome \\
\hline 1 & 73 & M & $\mathrm{Sq}$ & Chemo & 59 & 18 & \multirow{12}{*}{+} & $\mathrm{D}$ \\
\hline 2 & 67 & M & $\mathrm{Sm}$ & Chemo & 45 & $0^{*}$ & & $\mathrm{R}$ \\
\hline 3 & 48 & $\mathrm{M}$ & $\mathrm{Sq}$ & Chemo & 60 & 30 & & D \\
\hline 4 & 61 & M & $\mathrm{Sm}$ & Chemo & 60 & 5 & & $\mathrm{R}$ \\
\hline 5 & 60 & $\mathrm{M}$ & $\mathrm{Sq}$ & Chemo & 59 & 20 & & D \\
\hline 6 & 73 & M & $\mathrm{Sq}$ & Chemo & 60 & 90 & & $\mathrm{R}$ \\
\hline 7 & 66 & M & $\mathrm{Sq}$ & Chemo & 60 & 120 & & $\mathrm{R}$ \\
\hline 8 & 69 & M & $\mathrm{Sq}$ & Chemo & 48 & $0^{*}$ & & $\mathrm{R}$ \\
\hline 9 & 63 & M & $\mathrm{Sq}$ & Chemo & 57 & 17 & & $\mathrm{D}$ \\
\hline 10 & 65 & $\mathrm{M}$ & $\mathrm{Sq}$ & Chemo & 60 & 4 & & $\mathrm{R}$ \\
\hline 11 & 65 & $\mathrm{M}$ & $\mathrm{Ad}$ & Chemo & 60 & 20 & & $\mathrm{R}$ \\
\hline 12 & 69 & $\mathrm{M}$ & $\mathrm{Sm}$ & Chemo & 47 & 10 & & D \\
\hline $\operatorname{Mean} \pm \mathrm{s}$ & \pm 6 & & & & $56.3 \pm 5.9$ & $27.8 \pm 37.7$ & & \\
\hline
\end{tabular}

M: male; Sm: small cell carcinoma; Sq: squamous cell carcinoma; Ad: adenocarcinoma; Chemo: chemotherapy; BAL: bronchoalveolar lavage; D: died; R: recovered. *: pneumonitis developed during the radiotherapy. 

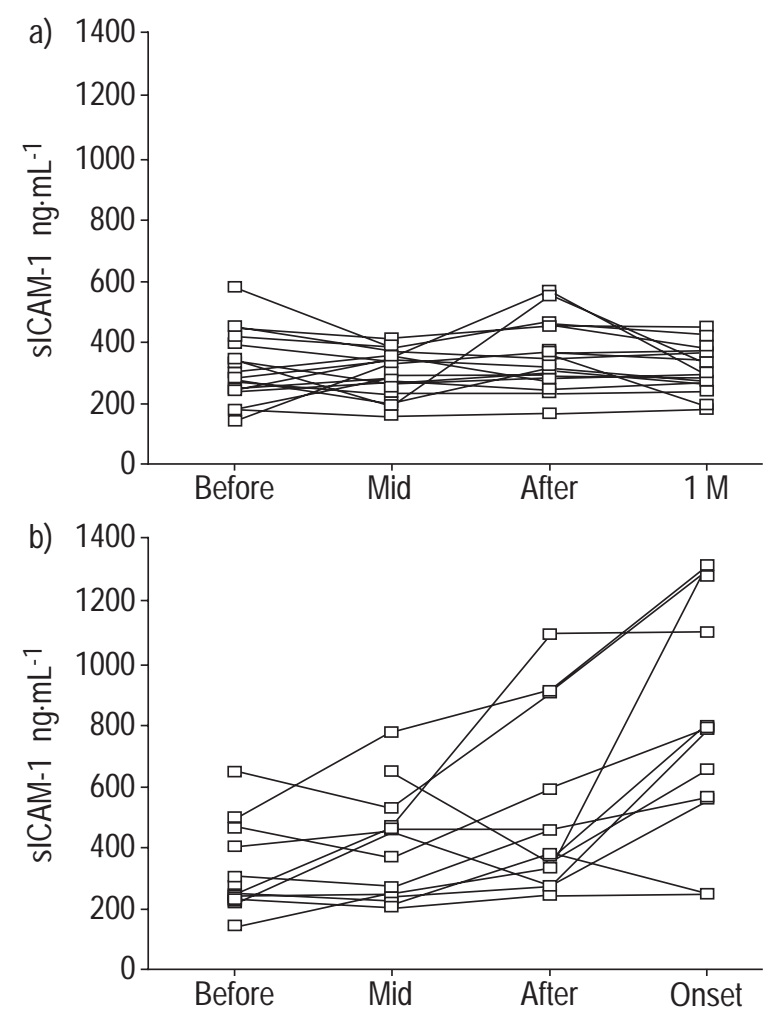

Fig. 1. - Change in soluble intercellular adhesion molecule-1 (sICAM1) levels in serum during radiotherapy in patients a) without and b) with pneumonitis. Blood samples were taken before, midway (Mid) and after radiotherapy. In the patients who developed radiation pneumonitis $(n=$ 12), additional blood samples were taken at the onset point (Onset). The mean \pm SD duration from the end of radiotherapy to the onset of radiation pneumonitis was $27.8 \pm 37.7$ days. Therefore, in the control group ( $n=$ $18)$, additional blood samples were taken 1 month $(1 \mathrm{M})$ after the radiotherapy.

of direct tissue damage induced by radiotherapy [25], resulting in a sensitization of autoreactive lymphocyte clones which migrate to the lung and react with pulmonary tissues. In the present study, the patient with radiation pneumonitis for which BAL was performed showed a marked increase in the total cell count and the number of

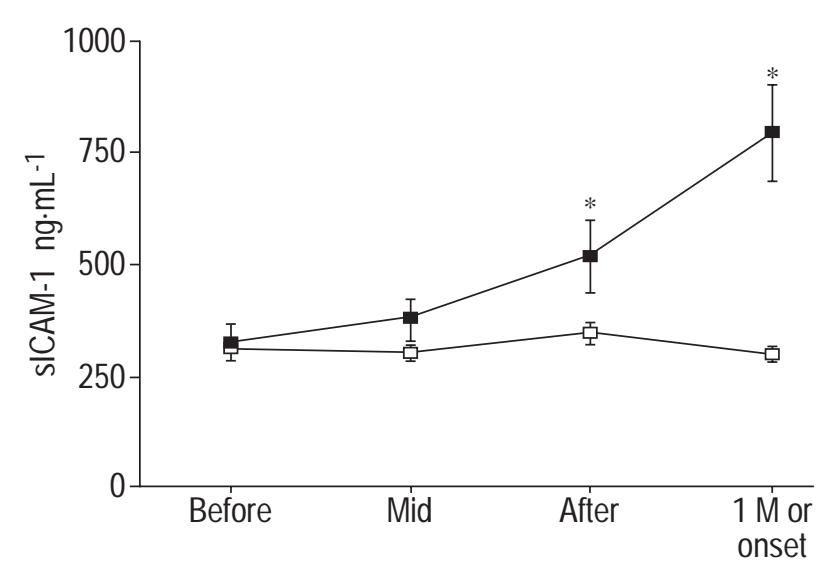

Fig. 2. - Change in the average soluble intercellular adhesion molecule1 (sICAM-1) concentration in serum during radiotherapy in patients with (ם) and without ( $\square$ ) pneumonitis. In the pneumonitis group, a significant elevation in sICAM-1 levels was seen at the end of radiation therapy and at the onset point of pneumonitis compared with a baseline "before" value, *: p<0.05. $1 \mathrm{M}$ : one month.
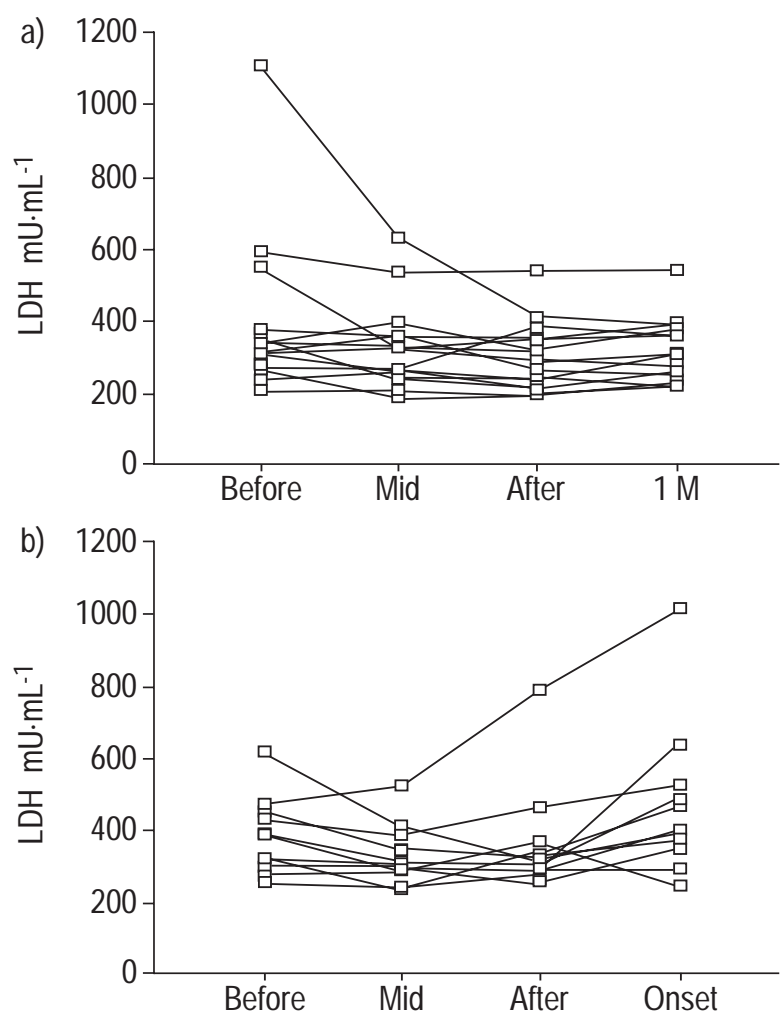

Fig. 3. - Change in lactate dehydrogenase (LDH) activity in serum during radiotherapy in patients a) without and b) with pneumonitis. Blood samples were taken before, midway (Mid) and after radiotherapy. In the patients who developed radiation pneumonitis $(n=12)$, additional blood samples were taken at the onset point (Onset). The mean \pm SD duration from the end of radiotherapy to the onset of radiation pneumonitis was $27.8 \pm 37.7$ days. Therefore, in the nonpneumonitis group $(n=18)$, additional blood samples were taken 1 month $(1 \mathrm{M})$ after radiotherapy.

lymphocytes. Histological studies based on an open lung biopsy taken from an irradiated field have also suggested lymphocytic infiltration as the dominant finding in acutephase pneumonitis [5]. Although the precise mechanism for radiation-induced lymphocytosis in the lungs remains uncertain, the participation of lymphocytes in the pathophysiology of radiation pneumonitis is apparent.

In the mechanism for the sequestration of leukocytes in the lungs, cell adhesion molecules play a key role. ICAM1 , which is expressed on various cell surfaces, including vascular endothelial cells, epithelial cells and lymphocytes [6], is a ligand for LFA-1, which is found on lymphocytes and other leukocytes [7]. The ICAM-1/LFA-1 interaction is important in T-lymphocyte activation and lymphocyte migration into inflammatory sites [8-11]. Recently, several investigators have reported on the role of ICAM-1 in the pathogenesis of radiation-induced injury. In in vitro studies, radiation induced the ICAM-1 expression on endothelial cells [12] and skin cultures [13]. In an animal model of radiation-induced lung injury, ICAM-1 expression of alveolar macrophages was significantly elevated after radiation [14]. Hallahan and Virudachlam [15] demonstrated that radiation-increased ICAM-1 expression in the pulmonary microvascular endothelium is accompanied by inflammatory cell infiltration in rat lungs, and that the i.v. administration of anti-ICAM-1 antibody blocks the radiation-induced leukocyte infiltration. They also indicated that radiation-induced leukocyte infiltration is not seen in 


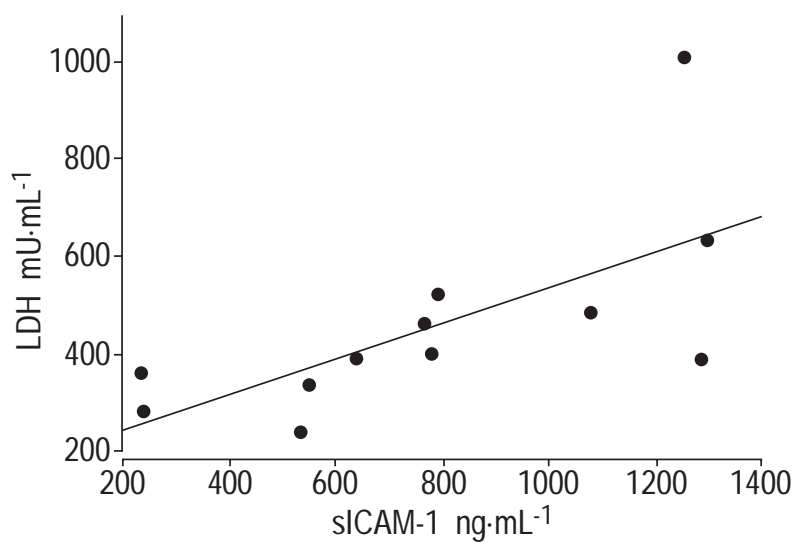

Fig. 4. - Correlation between soluble intercellular adhesion molecule-1 (sICAM-1) levels and lactate dehydrogenase (LDH) activity. There was a significant correlation between the serum sICAM-1 level and LDH activity only at the onset point of pneumonitis $(\mathrm{n}=12, \mathrm{r}=0.665, \mathrm{p}<0.02)$.

the lungs of ICAM-1-deficient mice [15]. In humans, it has been reported that radiation-induced ICAM-1-positive Tlymphocytes accumulate in the lungs [16]. These findings suggest that radiation can induce ICAM-1 expression on lung cells and that ICAM-1 is required for inflammatory cell infiltration into the irradiated lung.

ICAM-1 shed from cell surfaces exists as a soluble form in the blood and the alveolar lining fluid $[17,18]$. Measurement of SICAM-1 has been reported to be useful for evaluating and monitoring disease activity in various immunological and inflammatory disorders [18-24]. In pulmonary diseases with accompanying lymphocytic alveolitis, including hypersensitivity pneumonitis and sarcoidosis, sICAM-1 levels in the serum and BAL fluid are elevated and vary with disease activity [18]. Therefore, the measurement of sICAM-1 levels in serum and BAL fluid is useful not only for investigating the pathogenic mechanisms in lymphocytic alveolitis (especially in lymphocyte activation and emigration), but also for assessing the clinical status and disease activity. In the present study, sICAM-1 concentrations were measured in patients with radiation pneumonitis.

The early detection and differential diagnosis of radiation pneumonitis are important but sometimes difficult. Several factors, such as C-reactive protein (CRP) and LDH activity, have been examined as serum markers of radiation pneumonitis. Moreover, type III procollagen N-terminal peptides and K L-6, a mucin-like glycoprotein, have been reported to be sensitive markers [26]. In the present study, sICAM-1 levels were examined and compared with LDH activity. After radiotherapy, serum levels of sICAM-1 were elevated in the pneumonitis group. In some cases, sICAM1 levels began to increase at an early phase of irradiation. In contrast, serum levels of sICAM-1 did not increase from the baseline value during the radiotherapy in the nonpneumonitis group. Serum LDH activity showed a tendency to decrease in response to radiation therapy. This may imply that the decrease in LDH is caused by the cancer tissues. It is not until radiation pneumonitis develops that serum LDH activity increases. At this time, serum LDH activity may be derived from the damaged lung tissue. A significant correlation between serum sICAM-1 and LDH was seen only at the onset point of pneumonitis. From these findings, it appears that serum sICAM-1 may be a useful marker for the early detection of radiation pneumonitis and a more sensitive marker than LDH activity.

In the present study, the mean age of healthy controls was younger than that of irradiated patients with lung malignancy. Normal levels of SICAM-1 in serum decrease with age in children [27], but there has been no report on age-dependent changes of sICAM-1 levels in adults. Baseline levels of sICAM-1 in both irradiated groups were higher than the control values. The elevation is thought to be due to their malignancy, because an elevated concentration of serum sICAM-1 has been reported in malignancy [21], but not in infection [21]. Therefore, the sequential monitoring of sICAM-1, before and after radiotherapy, is thought to be important. In the pneumonitis group, the value at the time of onset was significantly higher than that before radiation.

In the BAL fluid from one patient with radiation pneumonitis, a marked increase in the number of lymphocytes and an elevation in sICAM-1 levels were observed. Although this was only one case, these findings suggest a possible relationship between the ICAM-1 expression induced by radiation and lymphocyte accumulation in the lungs. However, this increase in sICAM-1 levels in BAL fluid was not so prominent and some patients without pneumonitis also exhibited a comparable increase in sICAM-1 levels in BAL fluid. As the study population for which BAL was performed was small, the relationship
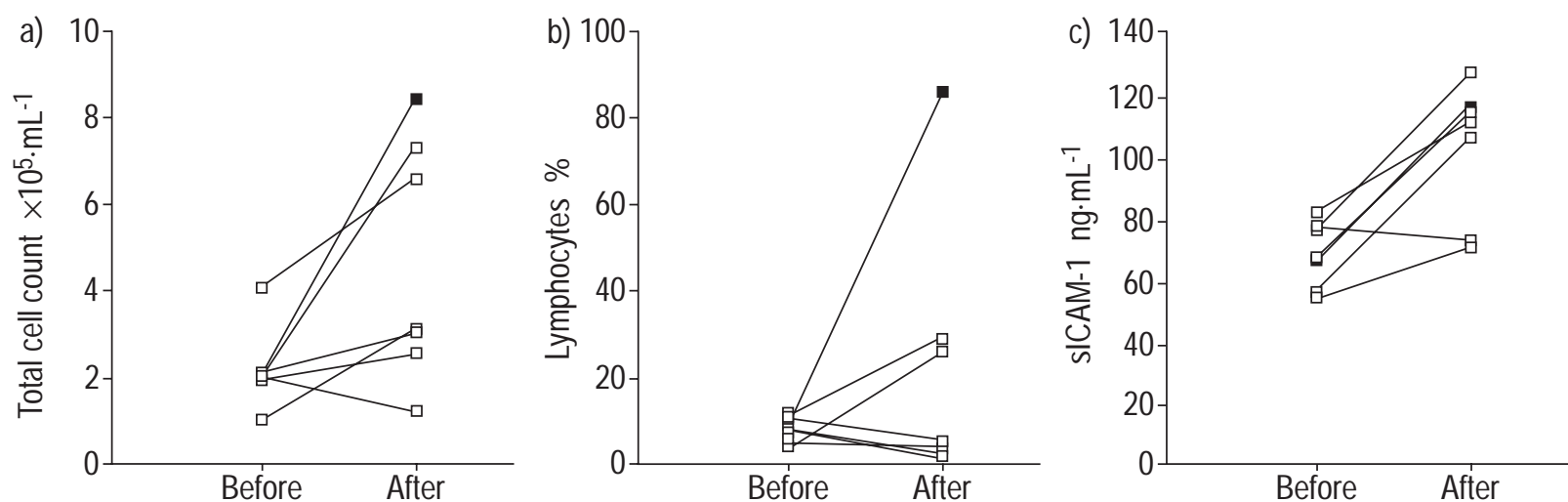

Fig. 5. - Changes in a) total cell count, b) percentage of lymphocytes and c) soluble intercellular adhesion molecule-1 (sICAM-1) levels in bronchoalveolar lavage (BAL) fluid after radiotherapy. BAL was performed before and after radiotherapy in six cases who did not develop radiation pneumonitis ( $\square$ ) and in one case who developed pneumonitis ( $\square$ ). 
between sICAM-1 levels and lymphocyte count in the BAL fluid remains unclear. It is likely that sICAM-1 levels in serum, which reflect the ICAM-1 expression in microvascular endothelial cells, but not in BAL fluid, may correlate with lymphocyte accumulation in the irradiated lungs. Furthermore, two of the patients without pneumonitis had mild lymphocytosis in the BAL fluid. Even when apparent radiation pneumonitis does not develop, the localized radiation-induced lung inflammation (with lymphocyte accumulation) may progress insidiously and subclinically. In such cases, even if localized expression of ICAM-1 occurs, it may not be reflected in the systemic sICAM-1 levels. To clarify the role of ICAM-1 in radiation-induced lung inflammation, it is necessary to carry out BAL in a greater number of cases.

From the results of the present study, the mechanism by which sICAM-1 increases at the onset of radiation pneumonitis remains unclear. A reactive oxygen species-mediated mechanism may be involved in ICAM-1 expression, because radiation causes an oxidative stress by producing oxygen radicals [28], which can stimulate the expression of adhesion molecules in the tissues [29].

In summary, intercellular adhesion molecule-1 may play an important pathophysiological role in the development of radiation pneumonitis, and soluble intercellular adhesion molecule-1 may be a useful marker for the early detection of radiation pneumonitis.

\section{References}

1. Gross NJ. Pulmonary effects of radiation therapy. Ann Intern Med 1977; 86: 81-92.

2. Roberts CM, Foulcher E, Zaunders JJ, et al. Radiation pneumonitis: a possible lymphocyte-mediated hypersensitivity reaction. Ann Intern Med 1993; 118: 696-700.

3. Cordier JF, Mornex JF, Lasne Y, et al. Bronchoalveolar lavage in radiation pneumonitis. Bull Eur Physiopathol Respir 1984; 20: 369-374.

4. Rosiello RA, Merrill WW, Rockwell S, et al. Radiation pneumonitis. Bronchoalveolar lavage assessment and modulation by a recombinant cytokine. Am Rev Respir Dis 1993; 148: 1671-1676.

5. Mark EJ. Lung Biopsy Interpretation. Baltimore, MD, Williams \& Wilkins, 1984; pp. 73-74.

6. Dustin ML, Rothlein R, Bhan AK, Dinarello CA, Springer TA. Induction by IL- 1 and interferon- $\gamma$. Tissue distribution, biochemistry, and function of a natural adherence molecule (ICAM-1). J Immunol 1986; 137: 245-254.

7. Marlin SD, Springer TA. Purified intercellular adhesion molecule 1 (ICAM-1) is a ligand for lymphocyte functionassociated antigen-1 (LFA-1). Cell 1987; 51: 813-819.

8. Neumayer HP, Schulz TF, Peters JH, Dierich MP. Importance of ICAM-1 for accessory cell function of monocytic cells. Immunobiology 1990; 180: 458-466.

9. Van Seventer GA, Shimizu Y, Horgan KJ, Shaw S. The LFA-1 ligand ICAM-1 provides an important costimulatory signal for $\mathrm{T}$ cell receptor-mediated activation of resting human T cells. J Immunol 1990; 144: 4579-4586.

10. Butcher EC. Leukocyte-endothelial cell recognition: three (or more) steps to specificity and diversity. Cell 1991; 67: $1033-1036$.

11. Oppenheimer-Marks N, Davis LS, Bogue DT, Ramberg J, Lipsky PE. Differential utilization of ICAM-1 and VCAM-1 during the adhesion and transendothelial mig- ration of human T lymphocytes. J Immunol 1991; 147 : 2913-2921.

12. Hallahan D, Kuchibhotla J, Wyble C. Cell adhesion molecules mediate radiation-induced leukocyte adhesion to the vascular endothelium. Cancer Res 1996; 56: 5150-5155.

13. Behrends U, Peter RU, Hintermeier-Knabe R, et al. Ionizing radiation induces human intercellular adhesion molecule-1 in vitro. JInvest Dermatol 1994; 103: 726-730.

14. Kawana A, Shioya S, Katoh H, Tsuji C, Tsuda M, Ohta Y. Expression of intercellular adhesion molecule-1 and lymphocyte function-associated antigen-1 on alveolar macrophages in the acute stage of radiation-induced lung injury in rats. Radiat Res 1997; 147: 431-436.

15. Hallahan DF, Virudachlam S. Intercellular adhesion molecule-1 knockout abrogates radiation induced pulmonary inflammation. Proc Natl Acad Sci USA 1997; 94: 64326437.

16. Nakayama Y, Makino S, Fukuda Y, Min K, Shimizu A, Ohsawa N. Activation of lavage lymphocytes in lung injuries caused by radiotherapy for lung cancer. Int $J$ Radiation Oncology Biol Phys 1996; 34: 459-467.

17. Rothlein R, Mainolfi EA, Czajkowski M, Marlin SD. A form of circulating ICAM-1 in human serum. J Immunol 1991; 147: 3788-3793.

18. Ishii Y, Kitamura S. Elevated levels of soluble ICAM-1 in serum and BAL fluid in patients with active sarcoidosis. Chest 1995; 107: 1636-1640.

19. Harning R, Mainolfi E, Bystryn JC, Henn M, Merluzzi JV, Rothlein R. Serum levels of circulating intercellular adhesion molecule 1 in human malignant melanoma. Cancer Res 1991; 51: 5003-5005.

20. Altomonte M, Colizzi F, Esposito G, Maio M. Circulating intercellular adhesion molecule 1 as a marker of disease progression in cutaneous melanoma. $N$ Engl J Med 1992; 327: 959.

21. Tsujisaki M, Imai K, Hirata $\mathrm{H}$, et al. Detection of circulating intercellular adhesion molecule-1 in malignant diseases. Clin Exp Immunol 1991; 81: 3-8.

22. Adams DH, Mainolfi E, Burra P, et al. Detection of circulating intercellular adhesion molecule- 1 in chronic liver diseases. Hepatology 1992; 16: 810-814.

23. Hashimoto S, Imai K, Kobayashi T, et al. Elevated levels of soluble ICAM-1 in sera from patients with bronchial asthma. Allergy 1993; 48: 370-372.

24. Seth R, Raymond FD, Makgoba MW. Circulating ICAM1 isoforms: diagnostic prospects for inflammatory and immune disorders. Lancet 1991; 338: 83-84.

25. Roswit B, White DC. Severe radiation injury of the lung. Am J Roentgenol 1977; 129: 127-136.

26. Kohno N, Hamada H, Fujioka S, Hiwada K, Yamakido M, Akiyama M. Circulating antigen KL-6 and lactate dehydrogenase for monitoring irradiated patients with lung cancer. Chest 1992; 102: 117-122.

27. Nash MC, Wade AM, Shah V, Dillon MJ. Normal levels of soluble E-selectin, soluble intercellular adhesion molecule-1 (sICAM-1), and soluble vascular cell adhesion molecule 1 (sVCAM-1) decrease with age. Clin Exp Immunol 1996; 103: 167-170.

28. Fraser RG, Pare JAP, Genereux GR. Diagnosis of Diseases of the Chest, 3rd Edn. Philadelphia, PA, WB. Saunders 1991; pp. 2551-2564.

29. Weber C, Erl W, Pietsch A, Strobel M, Ziegler-Heitbrock HLM, Weber PC. Antioxidants inhibit monocyte adhesion by suppressing nuclear factor-kB mobilization and induction of vascular cell adhesion molecule-1 in endothelial cells stimulated to generate radicals. Arterioscler Thromb 1994; 14: 1665-1673. 